This item was submitted to Loughborough's Research Repository by the author.

Items in Figshare are protected by copyright, with all rights reserved, unless otherwise indicated.

\title{
Measuring performance in the interpretation of chest radiographs: a pilot study
}

PLEASE CITE THE PUBLISHED VERSION

http://dx.doi.org/10.1016/j.crad.2016.11.004

PUBLISHER

(C) Elsevier on behalf of The Royal College of Radiologists

\section{VERSION}

AM (Accepted Manuscript)

\section{PUBLISHER STATEMENT}

This work is made available according to the conditions of the Creative Commons Attribution-NonCommercialNoDerivatives 4.0 International (CC BY-NC-ND 4.0) licence. Full details of this licence are available at: https://creativecommons.org/licenses/by-nc-nd/4.0/

\section{LICENCE}

CC BY-NC-ND 4.0

\section{REPOSITORY RECORD}

Chen, Yan, Jonathan James, Leng Dong, and Alastair G. Gale. 2019. "Measuring Performance in the Interpretation of Chest Radiographs: A Pilot Study”. figshare. https://hdl.handle.net/2134/24702. 


\section{Clinical Radiology}

\section{Measuring Performance in the Interpretation of Chest Radiographs - a Pilot Study \\ --Manuscript Draft--}

Manuscript Number:

Full Title:

Article Type:

Corresponding Author:
CRAD-D-16-00381R2

Measuring Performance in the Interpretation of Chest Radiographs - a Pilot Study

Original Paper

Yan Chen, PhD

Loughborough University

Loughborough, UNITED KINGDOM

Corresponding Author Secondary

Information:

Corresponding Author's Institution:

Loughborough University

Corresponding Author's Secondary Institution:

First Author:

Yan Chen, PhD

First Author Secondary Information:

Order of Authors:

Yan Chen, PhD

Jonathan James, Consultant Radiologist

Leng Dong

Alastair G Gale, Professor

Order of Authors Secondary Information:

Abstract:

Aim: To develop a system to assess the image interpretation performance of radiologists in identifying signs of malignancy on chest radiographs.

Materials and Methods: A test set of 30 chest radiographs was chosen by an experienced radiologist consisting of 11 normal and 19 abnormal cases. The malignant cases all had biopsy proven pathology; the normal and benign cases all had at least two years of imaging follow-up. Fourteen radiologists with a range of experiences were recruited. Participants individually read the test set displayed on a standard reporting workstation, with their findings entered directly onto a laptop running specially designed reporting software. For each case, relevant clinical information was given and the reader was asked to mark any perceived abnormality and rate their level of suspicion on a 5 point scale (normal, benign, indeterminate, suspicious or malignant). On completion, participants were given instant feedback with performance parameters including sensitivity and specificity automatically calculated. Opportunity was then given to review the cases together with an expert opinion and pathology. The time each participant took to complete the test was recorded.

Results: Six consultant radiologists who took part showed significantly better performance as determined by ROC analysis compared to eight specialist registrars ( $A \cup C=0.9297$ and 0.7648 respectively, $p=0.003$ ). There was a significant correlation with years of experience in the interpretation of chest radiographs and performance on the test set $(r=0.573, p=0.032)$. Consultant Radiologists completed the test significantly more quickly that the Specialist Registrars - mean time 19.65 minutes compared to 26.51 minutes $(p=0.033)$.

Conclusion: It is possible to use a test set to measure individual differences in the interpretation of chest radiographs. This has the potential to be a useful tool in performance testing. 


\section{Measuring Performance in the Interpretation of Chest Radiographs - a Pilot Study}

Yan Chen ${ }^{1}$, Jonathan J James ${ }^{2}$, Leng Dong ${ }^{1}$ \& Alastair G Gale ${ }^{1}$

1. Applied Vision Research Centre, Loughborough University, Loughborough, UK.

2. Nottingham Breast Institute, Nottingham University Hospitals, Nottingham, UK

Corresponding Author:

Yan Chen

Applied Vision Research Centre, Loughborough University, Loughborough, UK. LE11 3TU

Tel: 01509635739

Email: y.chen@lboro.ac.uk

Acknowledgements: With thanks to all the readers who took part in the study at the Nottingham Breast Institute. 


\section{Author Contributions}

1. Guarantor of integrity of entire study: Yan Chen

2. Study Concepts and Design: Yan Chen, Jonathan J James, Leng Dong, Alastair G Gale

3. Literature Research: Yan Chen, Jonathan J James

4. Clinical Studies: N/A

5. Experimental studies/data analysis: Leng Dong, Yan Chen

6. Statistical analysis: Leng Dong, Yan Chen

7. Manuscript Preparation: Yan Chen, Jonathan J James, Alastair G Gale

8. Manuscript Editing: Yan Chen, Jonathan J James, Alastair G Gale 


\section{Measuring Performance in the Interpretation of Chest Radiographs - a Pilot Study}

\section{Abstract}

Aim: To develop a system to assess the image interpretation performance of radiologists in identifying signs of malignancy on chest radiographs.

Materials and Methods: A test set of 30 chest radiographs was chosen by an experienced radiologist consisting of 11 normal and 19 abnormal cases. The malignant cases all had biopsy proven pathology; the normal and benign cases all had at least two years of imaging follow-up. Fourteen radiologists with a range of experiences were recruited. Participants individually read the test set displayed on a standard reporting workstation, with their findings entered directly onto a laptop running specially designed reporting software. For each case, relevant clinical information was given and the reader was asked to mark any perceived abnormality and rate their level of suspicion on a 5 point scale (normal, benign, indeterminate, suspicious or malignant). On completion, participants were given instant feedback with performance parameters including sensitivity and specificity automatically calculated. Opportunity was then given to review the cases together with an expert opinion and pathology. The time each participant took to complete the test was recorded.

Results: Six consultant radiologists who took part showed significantly better performance as determined by ROC analysis compared to eight specialist registrars ( $A U C=0.9297$ and 0.7648 respectively, $p=0.003)$. There was a significant correlation with years of experience in the interpretation of chest radiographs and performance on the test set $(r=0.573, p=0.032)$. Consultant Radiologists completed the test significantly more quickly that the Specialist Registrars - mean time 19.65 minutes compared to 26.51 minutes $(p=0.033)$.

Conclusion: It is possible to use a test set to measure individual differences in the interpretation of chest radiographs. This has the potential to be a useful tool in performance testing. 


\section{Introduction}

2 In recent years there has been considerable interest in generating individual performance data in the NHS. An assessment of individual performance is part of the appraisal and revalidation process

4 for all doctors in the UK and can be used to improve services, spot problems early and inform the 5 public.

6 Radiology is not immune to scrutiny and so consideration needs to be given to methods of assessing

7 performance in the different sub-specialities [1,2]. Performance measures might include those derived from real-life data generated from individual clinical practice, a peer review process where the clinical work of one radiologist is reviewed by another and from batches of test cases. In the UK, the NHS Breast Screening Programme (NHSBSP) successfully uses a test set based self-assessment scheme to generate individual performance data that can be compared across the programme. The Personal Performance in Mammographic Screening (PERFORMS) scheme has been running for over 25 years and is currently undertaken by over 800 readers each year. A batch of 60 mammograms, consisting of a mixture of normal and abnormal studies are made available twice a year. Each case is viewed and interpreted by the reader with decisions entered onto a password protected website. At the end of the reading session the individual gets immediate feedback of performance compared to an 'expert opinion' generated from a panel of experienced breast radiologists. When all the readers have undertaken the test detailed anonymised feed-back is produced enabling an individual to see how their performance compares to their peers nationally.

Chest radiographs are one of the most commonly requested and reported imaging investigations worldwide and the first line imaging test for patients with chest symptoms suspicious of malignancy. As with mammography, the ability to spot the early and potentially subtle signs of malignancy is crucial. Missed lung cancer on the chest radiograph is a very common source of error and cases are commonly encountered at error and discrepancy meetings [3-5]. 
The aim of this pilot study was to develop a similar system to the PERFORMS scheme for mammographic interpretation to assess the performance of radiologists in identifying signs of malignancy on chest radiographs.

\section{Materials and Methods}

A test set of 30 digital chest radiographs was chosen by a radiologist $(\mathrm{JJJ})$ with 13 years experience of chest radiograph interpretation at consultant level, who also provided the expert opinion on each case. The case mix for the study consisted 19 abnormal cases and 11 normal cases. The abnormal cases consisted of 16 biopsy proven malignant lesions and 3 benign cases. The benign cases all had features which were judged to be abnormal on the chest radiograph at the time of clinical presentation warranting further investigation - one was a case of multiple nodules (chicken pox pneumonia on CT and follow up) and two were solitary masses (a biopsy proven sclerosing haemangioma, and an area of inflammation which resolved on follow up). All the malignant cases had biopsy proven pathology; the normal and benign cases also had at least two years of follow up. All images were available as anonymised DICOM files.

Fourteen radiologists took part in the pilot study. Written consent was obtained from all participating radiologists (ethical approval was not deemed necessary following discussion with the local committee). There were six consultant radiologists and eight radiology specialist registrars. The consultant radiologists experience ranged from $11-26$ years (mean 17.8 years) and the registrars experience ranged from 1-5 years (mean 2.5 years).

Participants individually examined each case on a standard clinical workstation in a darkened reporting room with controlled ambient lighting. The usual image manipulation tools were available and relevant clinical information was provided. Findings were entered directly onto a laptop running specially designed reporting software adapted from the PERFORMS scheme. The participant 
was asked to mark any perceived abnormality on a low resolution version of the image on the laptop and rate their level of suspicion on a 5-point scale - Normal, Benign, Indeterminate, Suspicious or Malignant. The participants were instructed that a rating of Indeterminate, Suspicious or Malignant meant that referral for further investigation was required. A Normal or Benign rating meant no further action was needed. Each participant read the test set in a random order which was generated by the reporting software. Once the reporting software had identified the next case for reading this was selected from the worklist displayed on the reporting workstation. The time taken for each participant to complete the test was recorded.

On completion of the test set, each participant was given instant feedback on their individual performance. Five personal performance parameters were calculated automatically by the software and immediately available to view. There were two measures of sensitivity - cancer detection rate (CD) and a correct recommendation for further investigation (CR). This latter measure was used to take account of the cases that have an abnormality on the chest radiograph judged to require further assessment which were subsequently found to be benign. Specificity (CS), defined as a correct recommendation for no further investigation, was also calculated as was a positive and negative predictive value (PPV and NPV). If an individual's result disagreed with the expert radiology opinion this was highlighted. An opportunity was given to review any of the cases again, this time with the expert radiology opinion available to view and histopathology provided where applicable. After completing the test set and reviewing the results, participants were asked to fill in a questionnaire to provide feedback on the test itself and the educational potential.

Participants' overall performance was examined by performing Receiver Operating Characteristic (ROC) curve analysis, with areas under the curve (AUC) calculated. The results were analysed for the two groups of readers - consultant radiologists and specialist registrars. ANOVA, Bonferroni post hoc 
of any difference in the performance parameters between the groups with a $p$ value of $<0.05$ considered significant.

\section{Results}

The performance of the Consultant Radiologists and Specialist Registrars is shown in Figure 1. The performance of the consultant group as determined by ROC analysis was significantly better than the specialist registrars ( $A \cup C=0.9297$ and 0.7648 respectively, $p=0.003$ ).

Figure 2 shows the performance attributes for Consultant Radiologists and Specialist Registrars. There was a trend for superior performance by Consultant Radiologists across all the measures. Consultant Radiologists PPV and NPV was significantly better than the Specialist Registrars $(p=0.021$ and $p=0.045$ respectively).

There was a significant correlation with years of experience in the interpretation of chest radiographs and test set performance. This was determined by a positive correlation between years of experience and the AUC $(r=0.573, p=0.032)$ for each of the 14 individuals. A scatterplot summarizes the results (Figure 3). For Specialist Registrars as a group there was a significant association between experience and performance $(p=0.011)$. Consultant Radiologists as a group showed no significant association between experience and performance. Most participants finished the test within 30 minutes. Figure 4 shows the mean completion times of the test set for both groups, along with the standard errors. Consultant Radiologists completed the test significantly more quickly than the Specialist Registrars - mean time 19.42 minutes $(95 \% \mathrm{Cl}$ [15.94-22.90]compared to 27.71 minutes $(95 \% \mathrm{Cl}[18.32-37.10](p=0.0397)$. purposes. Suggestions for improvement included making prior images available and supplying more 
demographic data. All the participants found the ability to review the cases along with an expert opinion once the test set had been completed useful.

\section{Discussion}

Errors will occur in any visual inspection task however expert the reader [5]. The measurement of individual performance is potentially an important tool in radiology. It has the potential to educate and improve the quality of interpretations, to spot problems early reducing potential harms and provide evidence for the individual, institution and public of a safe and effective service. In an ideal world we would be able to extract suitable performance measures from an individual's everyday reporting practice. Such information even when it can be generated is useful but it does not allow comparisons between individuals and institutions where case mix is different. Obtaining a true reflection of individual performance is also difficult when the incidence of abnormalities is relatively low and patients are not followed up. For instance, it may take several years before interpretation errors become apparent when a patient presents with more advanced disease. The use of test sets provides a solution and this pilot study demonstrates that it would be possible to develop a performance testing scheme for the interpretation of chest radiographs along the lines of that used successfully for mammography interpretation for the last 25 years.

The results show it has been possible to measure performance differences between individuals. Consultant Radiologists showed significantly better performance compared to specialist registrars. The use of test sets to measure performance in the interpretation of chest radiographs is not new and others have also found that improved performance is associated with experience $[6,7]$. Similarly in the PERFORMS scheme for mammographic interpretation, performance is significantly related to years of reading experience [8]. In our study only radiologist performance was measured, but others 
have used test sets to measure performance between different professional groups $[6,7,9]$. For instance, Monnier-Cholley et al showed similar performance between radiologists and chest physicians in the interpretation of chest radiographs, with both groups being significantly better than anaesthetists [7].

It is important for any performance testing system to measure sensitivity and specificity. There are two potential sources of interpretation error that the participant could make. The first is underreporting or the failure to spot the abnormality on the image. The second is over-reporting leading to the patient being referred for unnecessary additional tests which adds to costs and also increases patient anxiety. For this pilot study we have adapted the software used in PERFORMS which has evolved over the last 25 years as the mammographic interpretation testing scheme has matured. One of its strengths is the ability of each individual participant to obtain instant feedback, with key performance parameters including sensitivity and specificity instantly available. The software used in this test also requires the participant to mark the area which is considered abnormal. This avoids the situation where the participant may correctly score the case as abnormal but have actually misclassified a normal feature. Once the test is completed the software allows the participant to review either all the cases along with the expert opinion and pathology were available or just elect to review discrepant cases. Learning from the discrepancies can improve performance and the ability to review cases provides educational opportunities for the participant similar to those provided by error and discrepancy meetings and peer review processes [2,3]. The results of the post-test questionnaire complete by all our participants also confirmed the learning opportunities such schemes provide. 
143 If assessing performance with tests sets is to be acceptable it is important that taking part does not

144 require an excessive time commitment. In this study participants were able to work at their own

145 rate with no set time limit; most completed the test within 30 minutes. Interestingly the consultant

146 radiologists who showed the better performance also finished significantly more quickly. Similar

147 observations have also been noted in participants undertaking the PERFORMS scheme. Most readers will complete the PERFORMS set of 60 mammography studies in less than two hours. Those who completed the test within one and a half hours showed no difference in cancer detection but did show significantly better specificity [9].

One of the potential criticisms of using tests set to assess an individual is whether it is an accurate reflection of real life performance [10]. A test set by its very nature is enriched with abnormal cases and the incidence of cancers is significantly higher than it would be in everyday practice. Also if a study is flagged as being abnormal requiring further investigation the reader knows that no additional tests or investigations will be performed on the patient as they would be in real life. Consequently the participant is viewing and interpreting studies in an artificial environment which has the potential to interfere with image interpretation [11]. Data from the PERFORMS scheme correlation between the detection of small breast cancers in real life and the overall cancer detection rate in the PERFORMS set $[9,12]$. Work is needed to see if a similar relationship can be demonstrated between the real life interpretation of chest radiographs and performance in a test set.

In conclusion, this pilot study suggests that it is possible to measure individual performance in the interpretation of chest radiographs in a similar fashion to the PERFORMS scheme undertaken by 
167 readers of screening mammography in the UK. Clearly these findings need to be validated in a much 168 larger group of participants. There is already much interest in performance testing of individuals in 169 the NHS. In radiology the use of test sets to assess performance in a commonly requested and 170 interpreted investigation like the chest radiograph has the potential to be a useful tool. 
1) Augustine K, McCoubrie P, Wilkinson JR, McKnight L. Workplace-based assessment in radiology where to now? Clinical Radiology 2010;65:325-332

2) Strickland NH. Quality assurance in radiology: peer review and peer feedback. Clinical Radiology $2015 ; 70: 1158-1164$

3) The Royal College of Radiologists. Standards for learning from discrepancies meetings.

4) Quekel LG, Kessels AG, Goei R, Van Engelshoven JM. Miss rate of lung cancer on the chest radiograph in clinical practice. Chest 1999;115:720-724

5) Muhm JR, Miller WE, Fontana RS, Sanderson DR, Uhlenhopp MA. Lung cancer detected during a screening program using four-month chest radiographs. Radology 1983;148:609-615

6) Potchen EJ, Cooper TG, Sierra AE, Aben GR, Potchen MJ, Potter MG, Siebert JE. Measuring Performance in Chest Radiography. Radiology 2000;217:456-459

7) Monnier-Cholley L, Carrat F, Cholley BP, Tubiana J-M, Arrivé L. Detection of Lung Cancer on Radiographs: Receiver Operating Characteristic Analysis of Radiologists', Pulmonologists' and Anesthesiologists' Performance. Radiology 2004;233:799-805

8) Scott HJ, Gale AG. How much is enough: factors affecting the optimal interpretation of breast screening mammograms. In Jiang $Y$ and Sahiner $B$, eds. Image Perception, Observer Performance, and Technology Assessment. Proc SPIE 2007;6515

9) Gale A, Scott S. Measuring Radiology Performance in Breast Screening. In: Michell MJ, Reznek $\mathrm{RH}$, Husband JE, editors. Breast Cancer: Contemporary Issues in Cancer Imaging. Cambridge University Press; (2010) p. 29-45

10) Soh BP, Lee W, Kench PL, Reed WM, McEntee MF, Poulos A, Brennan PC. Assessing reader performance in radiology, an imperfect science: Lessons from breast screening. Clinical Radiology 2012;67:623-628 
11) Gur D, Bandos Al, Cohen CS et al. The "Laboratory" Effect: Comparing Radiologists' Performance and Variability during Prospective Clinical and Laboratory Mammography Interpretations. Radiology 2008;249:47-53

200

12) Scott HJ, Evans A, Gale AG et al. The relationship between real life breast screening and an annual self-assessment scheme. Proc SPIE 2009;7263

Figure 1. Overall performance of Specialist Registrars and Consultant Radiologists in the interpretation of the test set.

Figure 2. Mean performance measures shown with standard error.

209

Figure 3. Scatterplot demonstrating the relationship between AUC and years of experience.

210

Figure 4. Mean time to report the 30 case test-set.

211 


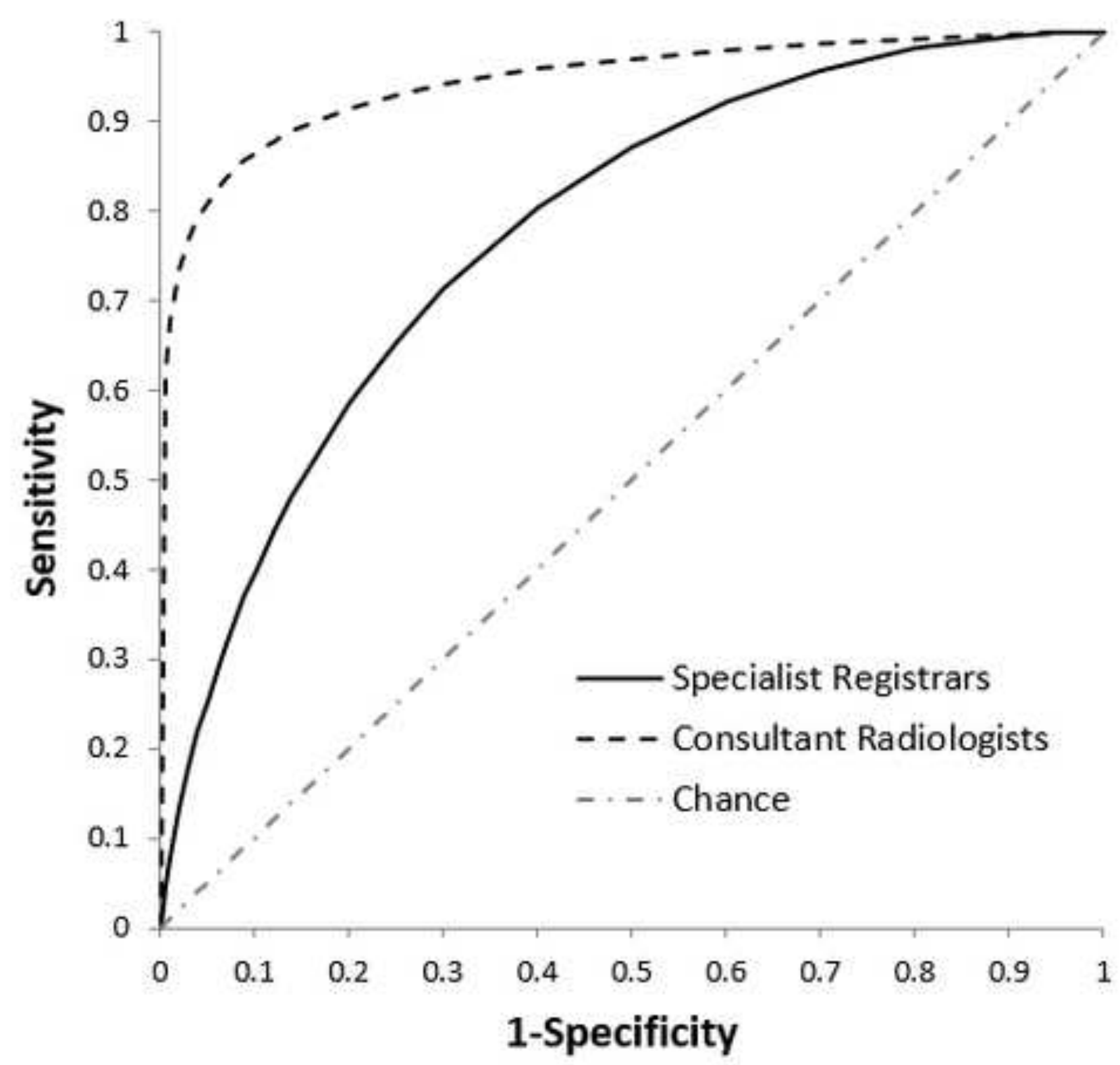

AUC for Consultant Radiologists $=0.9297$ \& AUC for Specialist Registrars $=0.7648, p=0.003$ 
- Specialist Registrars

E Consultant Radiologists

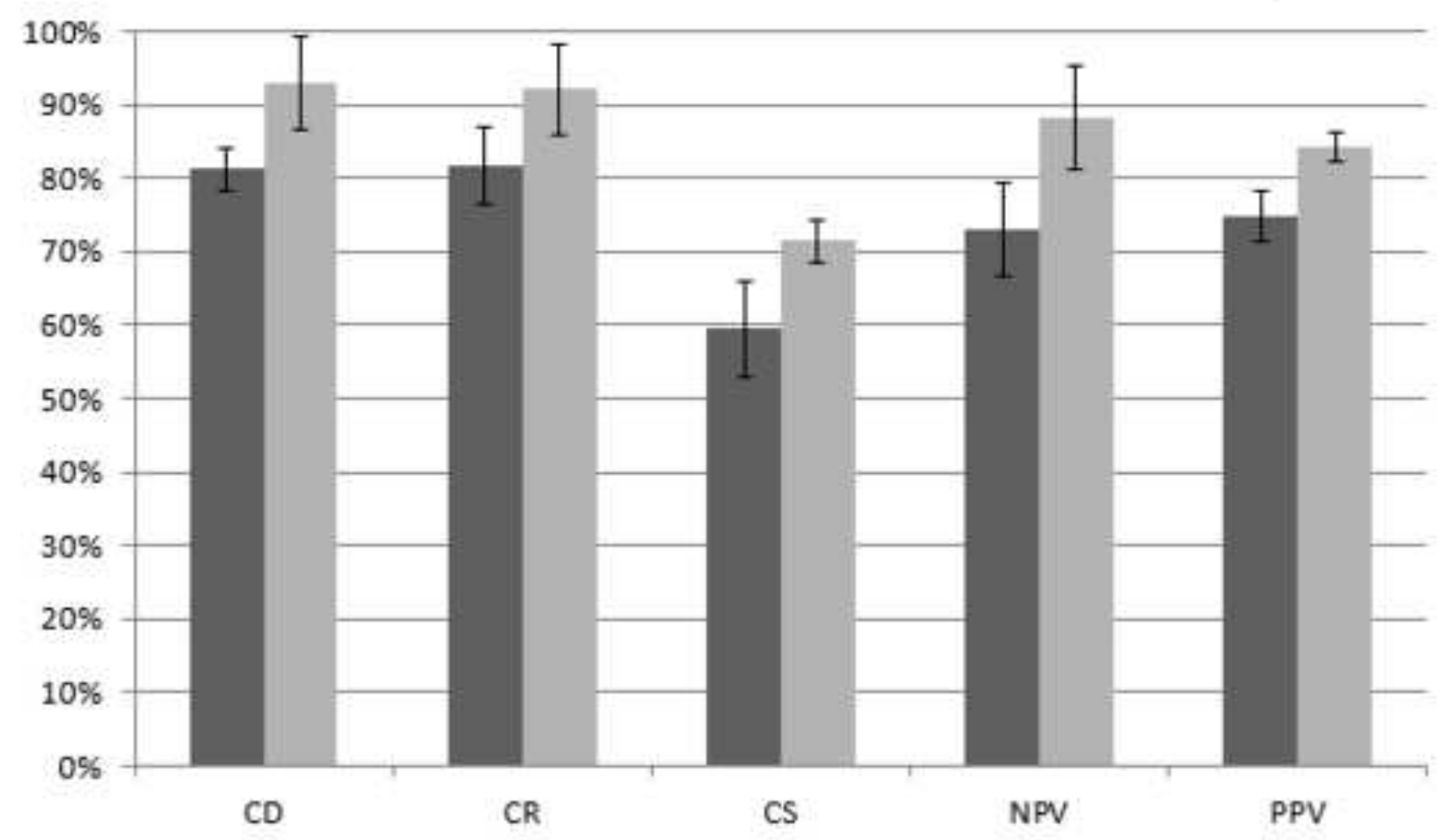

$C D$ - Cancer Detection - sensitivity $(p=0.062)$

$C R$ - Correct Referral for further investigation - sensitivity $(p=0.081)$

CS - Correct recommendation for no further investigation - specificity $(p=0.104)$

NPV - Negative Predictive Value $(p=0.045)$

PPV - Positive Predictive Value $(p=0.021)$ 


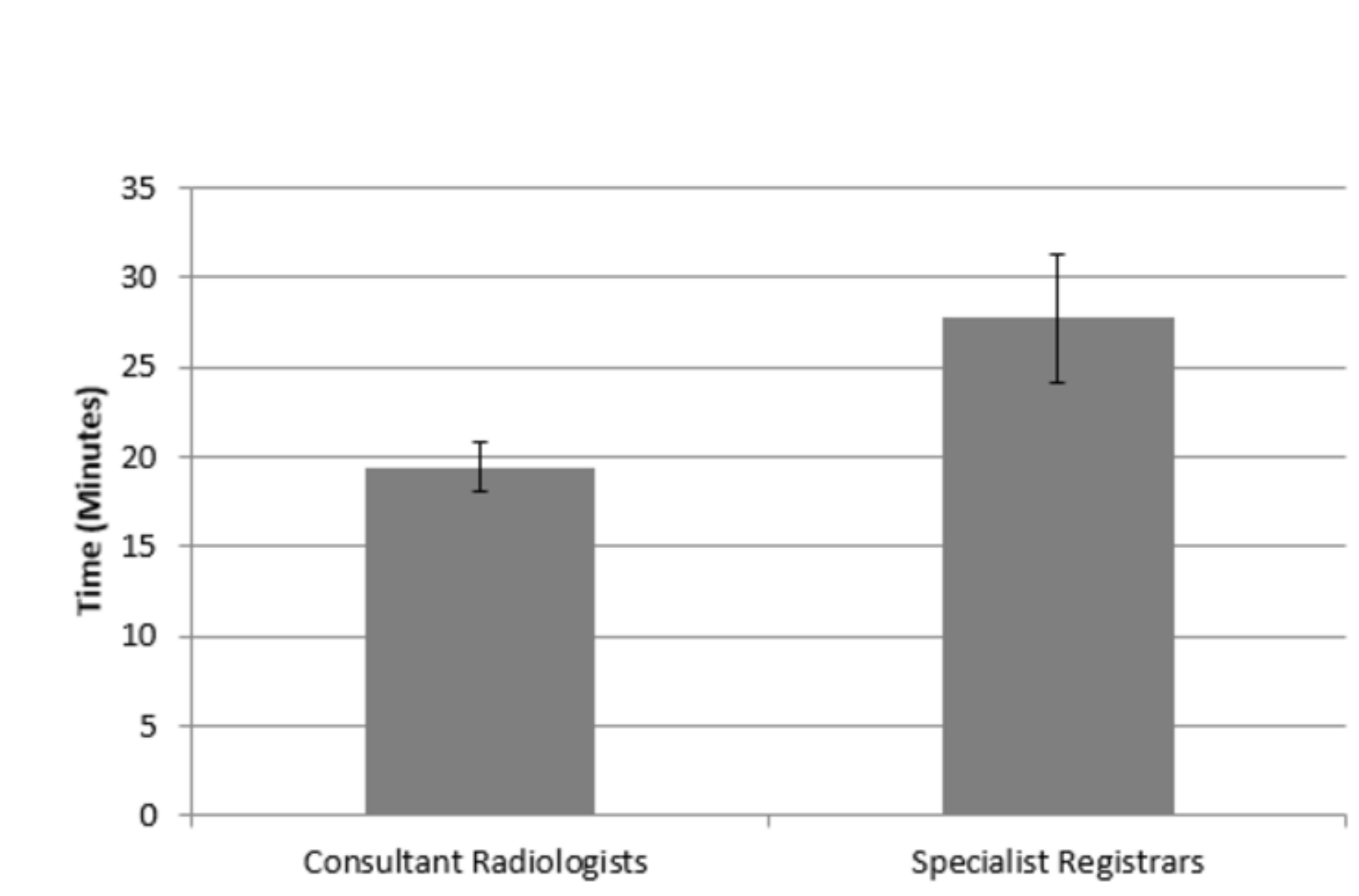




\section{Highlights}

- Consultant radiologists perform significantly better and also faster than radiology residents in chest radiography interpretation

- Test sets are a valid and practical tool for measuring performance differences.

- Test sets with immediate feedback are an excellent educational tool 